\title{
ON A CONJECTURE OF GRAHAM CONCERNING A SEQUENCE OF INTEGERS
}

\author{
BY \\ E. Z. CHEIN
}

Let $0<a_{1}<\cdots<a_{n}$ be integers and $(a, b)$ denotes the greatest common divisor of $a, b$. R. L. Graham [1] has conjectured that

$$
\frac{a_{i}}{\left(a_{i}, a_{j}\right)} \geq n
$$

for some $i$ and $j$. In a recent paper Weinstein [2] has improved Winterle's result [3] and has proven the following interesting theorem:

Theorem. (Weinstein). If $A$ is the sequence $a_{1}<\cdots<a_{n}$, where $a_{k}=P$, a prime for some $k$ and $P \neq\left(a_{i}+a_{j}\right) / 2,1 \leq i<j \leq n$, then

$$
\max _{i, j}\left\{\frac{a_{i}}{\left(a_{i}, a_{j}\right)}\right\} \geq n \text {. }
$$

In this paper we prove that the condition $P \neq\left(a_{i}+a_{j}\right) / 2$ in Weinstein's Theorem is unnecessary by modifying Weinstein's argument. We use Weinstein's notation throughout the paper. Our principal result is the following

THEOREM. If $A$ is the sequence $0<a_{1}<\cdots<a_{n}$, where $a_{k}=P$, a prime for some $k$, then

$$
\frac{a_{i}}{\left(a_{i}, a_{j}\right)} \geq n
$$

for some $i$ and $j$.

Proof. Assume there exists a sequence $A$, say $0<a_{1}<\cdots<a_{n}$, where $a_{k}=P$ for some $k$ and

$$
\frac{a_{i}}{\left(a_{i}, a_{j}\right)}<n
$$

for all $i$ and $j$.

Let $B$ be the subsequence $b_{1}<\cdots<b_{g}<\cdots<b_{r}$ of $A$ consisting of all terms of $A$ which are not divisible by $P$. By results of Winterle [3] and Vélez [4], the

I am greatly indebted to Professor Christine W. Ayoub and Mr. Patrick Wotus for their kind discussion of this problem.

Received by the editors September 9, 1977 and, in revised form, December 19, 1977. 
conjecture is true if $a_{1}$ is prime or $n-1$ is prime, so we can assume that neither $a_{1}$ nor $n-1$ is prime. Then we have

$$
b_{1}+(g-1) \leq b_{g}<P<n-1, \quad(g \geq 1)
$$

and so

$$
\frac{P}{b_{1}} \geq \frac{P}{p-g}>\frac{n-1}{n-g-1}
$$

This gives

$$
\begin{aligned}
\frac{(n-g-1) p}{(n-g-1) P} & \geq(n-g-1) \frac{P}{b_{1}} \geq(n-g-1) \frac{P}{p-g} \\
& >(n-g-1) \frac{n-1}{n-g-1}=n-1,
\end{aligned}
$$

but $(n-g-1) P /\left((n-g-1) P, b_{1}\right)$ is an integer, so is greater than or equal to $n$. Hence

$$
a_{i} \leq(n-g-2) P
$$

for all $a_{i} \in A \backslash B$. Also $P \nmid b_{i}$, so

$$
b_{r} \leq n-1
$$

We now define a mapping $T\left(b_{i}\right)$ for all $b_{i} \in B$ by

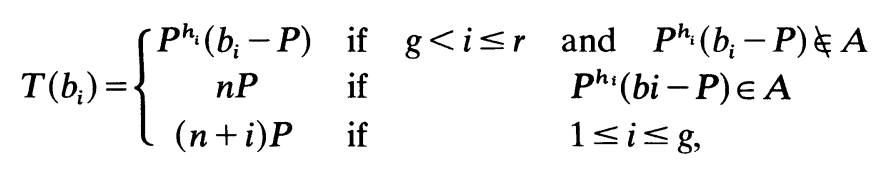

where $h_{i}$ is the largest non-negative integer such that $P^{h_{i}}\left(b_{i}-P\right) \leq(n-g-2) P$.

We next show that $T$ is $1-1$. If $1 \leq i \leq g$, it is clear that the $T\left(b_{i}\right)$ are all distinct. In the case $g<i \leq r$, since $b_{i} \leq n-1$ and $b_{1}+g \leq P$, it follows that $b_{i}-P \leq n-g-2$. Then $h_{i} \geq 1$ so that $P \mid T\left(b_{i}\right)$. Also, since $\left(P^{h_{i}}\left(b_{i}-P\right), b_{i}\right)=1$ we must have $T\left(b_{i}\right) /\left(T\left(b_{i}\right), b_{i}\right)=T\left(b_{i}\right)$. Now if $T\left(b_{i}\right) \leq n-g-2$, then $T\left(b_{i}\right) P \leq$ $(n-g-2) P$, which contradicts $(2)$, the definition of $T\left(b_{i}\right)$. So

$$
T\left(b_{i}\right) \notin A
$$

except possibly when $n-g-1 \leq T\left(b_{i}\right) \leq n-1$.

Now $P \mid T\left(b_{i}\right)$ and $1+g<b_{1}+g \leq P$. Since there is at most one term of $P$ consecutive integers which is divisible by $P$, we have

$$
\left|\left\{T\left(b_{i}\right) \mid \gamma \geq r \geq 1\right\} \cap A\right| \leq 1 .
$$

Now if $T\left(b_{i}\right)=T\left(b_{j}\right)$, then $P^{h_{i}}\left(b_{i}-P\right)=P^{h_{j}}\left(b_{j}-P\right)$, so $b_{i}=b_{j}$. Hence the $T\left(b_{i}\right)$ are distinct for all $i$, so that $T$ is $1-1$. 
We next define $F\left(a_{i}\right)$ for all $a_{i} \in A$ by

$$
F\left(a_{i}\right)=\left\{\begin{array}{ccc}
a_{i} & \text { if } & P \mid a_{i} \\
T\left(a_{i}\right) & \text { if } & P \nmid a_{i}
\end{array},\right.
$$

Then $P \mid F\left(a_{i}\right)$ for all $i$. In view of (1) and (2), $F\left(a_{i}\right) \neq F\left(a_{j}\right)$ if $i \neq j$, so $F$ is 1-1. From (1) and (2) we see that

$$
|A| \leq(n-g-2)+g+1=n-1,
$$

which contradicts the fact $|A|=n$. This completes the proof of our theorem.

\section{REFERENCES}

1. R. L. Graham, Unsolved problem 5749, Amer. Math. Monthly 77 (1970), 775.

2. G. Weinstein On a conjecture of Graham concerning greatest common divisors proceedings Amer. Math. Soc. 63 (1977), 33-38.

3. Riko Winterle, A problem of R. L. Graham in combinatorial number theory, Proc. Louisiana Conf. on Combinatorics, Graph Theory and Computing (Louisiana State Univ., Baton Rouge, La., 1970, pp. 357-361. MR 42 \#3051.

4. W. Y. Vélez, Some remarks on a number theoretic problem of Graham, Acta. Arith. 32 (1977), no. 3, 233-238.

\section{Department of Mathematics}

The Pennsylvania State University

University Park, Pennsylvania 16802 